\title{
Transatlantica
}

Revue d'études américaines. American Studies Journal

\section{La migration des personnes de couleur libres dans les Amériques et dans l'espace atlantique avant les abolitions de l'esclavage}

Université de Chicago à Paris, 4 octobre 2019

\section{Anne-Claire Faucquez}

\section{Q OpenEdition}

Journals

Édition électronique

URL : https://journals.openedition.org/transatlantica/13821

DOI : 10.4000/transatlantica.13821

ISSN : $1765-2766$

Éditeur

Association française d'Etudes Américaines (AFEA)

Référence électronique

Anne-Claire Faucquez, "La migration des personnes de couleur libres dans les Amériques et dans

l'espace atlantique avant les abolitions de l'esclavage », Transatlantica [En ligne], 1 | 2019, mis en ligne le 01 juin 2020, consulté le 01 février 2023. URL : http://journals.openedition.org/transatlantica/13821 ; DOI : https://doi.org/10.4000/transatlantica.13821

Ce document a été généré automatiquement le 1 février 2023

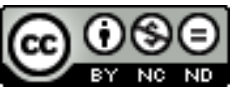

Creative Commons - Attribution - Pas d'Utilisation Commerciale - Pas de Modification 4.0 International - CC BY-NC-ND 4.0

https://creativecommons.org/licenses/by-nc-nd/4.0/ 


\section{La migration des personnes de couleur libres dans les Amériques et dans l'espace atlantique avant les abolitions de l'esclavage}

Université de Chicago à Paris, 4 octobre 2019

Anne-Claire Faucquez

\section{NOTE DE L'AUTEUR}

\section{Organisateurs:}

Claire Bourhis-Mariotti, maitre de conférences en histoire africaine américaine à l'Université Paris 8.

Lawrence Aje, maitre de conférences en histoire africaine américaine à l'Université Paul Valéry-Montpellier 3.

1 Cette journée internationale portant sur « la migration des personnes de couleur libres dans les Amériques et dans l'espace atlantique avant les abolitions de l'esclavage ", organisée par Claire Bourhis-Mariotti et Lawrence Aje, s'est déroulée à l'université de Chicago à Paris le 4 octobre 2019. Son objectif était d'établir un état des lieux de la recherche sur ces migrations dans un espace géographique très large, les Amériques et l'espace atlantique, et dans une période chronologique bien définie, avant les abolitions de l'esclavage (1834 dans l'empire britannique, 1865 aux États-Unis, 1886 à Cuba). À travers des anecdotes de vies personnelles ou des trajectoires de groupes, il s'agissait d'évaluer l'impact que la condition raciale et juridique particulière de ces migrants pouvait avoir sur leurs choix migratoires, leurs motivations et les conditions dans lesquelles ces circulations s'effectuaient. Si cet espace atlantique et caribéen pouvait représenter une source d'espoir, il pouvait également être lieu de désenchantement. En quoi cette histoire migratoire diffère-t-elle de celle des Blancs? Peut-on voir des 
tendances migratoires propres aux Africains-Américains qui justifieraient l'emploi du concept de "Black Atlantic History "? Ce sont à ces multiples interrogations que ces sept communications de doctorant.e.s et chercheurs.es confirmé.e.s, français et américains ont tenté de répondre.

2 La première session de la matinée s'est ouverte sur l'intervention de Seynabou Thiam (doctorante de l'université Paris 8), intitulée "La Migration des loyalistes noirs au Canada en 1783 : un passage contrôlé ? » Seynabou Thiam nous a rappelé que parmi les 60000 loyalistes restés fidèles à la couronne d'Angleterre après la guerre d'indépendance, $10 \%$ étaient des personnes de couleur. Ces dernières faisaient partie des 50000 à 100000 esclaves en fuite ayant rejoint les troupes britanniques suite à la déclaration du 7 novembre 1775 de Lord Dunmore. Or, si la signature du traité de Paris de 1783 stipulait que les prisonniers des deux camps devaient être libérés, les esclaves fugitifs ne pouvaient quant à eux être émancipés et devaient être ramenés à leurs maitres. La migration des Noirs depuis New York vers les Provinces maritimes du Canada fut donc hautement contrôlée et recensée dans un livre portant le nom de The Book of Negroes. Ce document est une source inestimable car il recense l'ensemble des noms, âges, descriptions physiques et destinations de ces migrants de couleur. Le petit village de Birchtown en Nouvelle-Ecosse reçut par exemple 3200 réfugiés noirs en 1784 . Cependant, même s'ils parvinrent à se constituer en communauté, centrée autour de l'église épiscopale et de ses écoles ouvertes par la Society for the Propagation of the Gospel (la branche missionnaire de l'Église anglicane), ces migrants de couleur furent soumis aux mêmes restrictions sociales et politiques qu'aux États-Unis et vivaient séparés des loyalistes blancs. Ils ne purent obtenir de terres et devinrent pour la plupart métayers, se virent privés de leurs droits civils, et, pour certains, furent même remis en esclavage. Ces désillusions économiques et sociales conduisirent à de nombreuses tensions, émeutes voire exils, notamment vers la Sierra Leone où 1196 libres de couleur se rendirent en 1792, suivis de 2000 nouveaux réfugiés en 1814. Seynabou Thiam conclut sur la lente reconnaissance de cette histoire africaine au Canada, qui ne fut commémorée qu'à partir de 1996 lors de la mise en place du Black History Month canadien.

3 Nathalie Dessens (professeur à l'université de Toulouse Jean Jaurès) amorce sa réflexion sur la malléabilité du concept d'histoire atlantique, laquelle permet un décloisonnement, une porosité entre les espaces coloniaux et facilite l'étude de la mobilité qui, à l'époque coloniale, ne concernait pas uniquement le groupe des libres de couleur. Ville dynamique à la croissance démographique constante (passant de 8000 à 102000 habitants entre 1803 en 1840), lieu de refuge des Acadiens ou des exilés de la révolution haïtienne et des guerres napoléoniennes, La Nouvelle-Orléans se trouvait au cœur des échanges avec Cuba, le Mexique, la Grande Colombie (actuelle République de Colombie) et la France, où ces émigrés se rendaient pour trouver une émulation intellectuelle et culturelle. Né à La Nouvelle-Orléans, Louis Charles Roudanez fit par exemple ses études de médecine en France avant de rentrer aux États-Unis, où il fonda le premier journal africain-américain du Sud. La famille Tinchant se démarquait également par l'étendue de son réseau commercial, vendant en Europe du tabac produit au Mexique.

4 Parmi la population extrêmement stratifiée de la ville, les Noirs libres constituaient une condition juridique intermédiaire (entre les Blancs et les esclaves). Ces derniers se voyaient privés de certains droits civiques mais bénéficiaient d'une totale liberté de 
mouvement. De même pouvaient-ils appartenir à la milice et posséder une propriété foncière. Si les Noirs libres étaient numériquement peu importants au XVIII siècle, l'arrivée des réfugiés suite à la révolution haïtienne (car à Haïti ils étaient considérés au même titre que l'ennemi blanc) tripla leur nombre et renforça leur groupe. De nombreux mariages eurent d'ailleurs lieu entre Louisianais et Dominguois. Ils jouèrent un rôle socio-économique, politique et culturel non négligeable, notamment par leurs capacités de mobilité identiques à celles des Blancs.

Insister sur ces circulations, ces allers-retours de part et d'autre de l'Atlantique et la constitution de ces réseaux permet de percevoir cet espace atlantique dans une perspective dynamique, comme un lieu d'échange. Les libres de couleur y ont ainsi joué un rôle primordial, revendiquant leurs droits et privilèges de classe, luttant pour l'abolition de l'esclavage (Charles Roudanez était présent à Paris en 1848), contribuant aux mouvements artistiques de l'époque (notamment par l'écriture militante de poèmes) et participant au recentrement de La Nouvelle-Orléans dans un Atlantique créole et dans une grande Caraïbe en plein essor.

Dans leurs deux communications, «Migrations and Missionaries: the Haitian Diaspora and "African" Christianity in Early America " et «King Cotton Exiles in Trinidad: Examining the formation of an African American Diaspora in the late 1830s ", Ronald Angelo Johnson (Associate Professor, Texas State University) et Ousmane PowerGreene (Associate Professor, Clark University, Worcester, MA) mettent en exergue la façon dont les migrations de Noirs libres en Haïti et à Trinidad dans les années 1830 ont pu façonner l'idée d'un nationalisme noir.

7 Ronald Angelo Johnson examine comment les Noirs libres en Haïti et aux États-Unis sont parvenus à forger une identité noire transnationale à la fois à travers les expériences des immigrants haïtiens aux États-Unis et des missionnaires noirs américains en Haïti. Il souligne le rôle prééminent des communautés catholiques et protestantes qui ont servi de canaux culturels et ont facilité l'assimilation des migrants de couleur.

8 Le début du XIX ${ }^{e}$ siècle a vu le renforcement conjoint du protestantisme noir dans les deux pays: tout d'abord par la politique des dirigeants de la nouvelle république haïtienne, qui souhaitaient évangéliser la population restée traditionnellement proche du catholicisme et du vaudou, ainsi que la naissance des premières Églises protestantes noires dans les grandes villes du Nord-Est des États-Unis, notamment à Philadelphie avec la Free African Society en 1787 ou la First African Baptist Church en 1788. La communication souligne l'impact considérable de l'immigration de réfugiés venant de Saint-Domingue en 1793 sur ces nouvelles Églises (Richard Allen et Absalom Jones quittèrent leurs Églises pour créer la Bethel African Episcopal Methodist Church et l'African Episcopal Church of St Thomas). Ces «nègres français" s'assimilèrent facilement dans la population de la ville. La famille Deveaux, installée à Savannah, joua également un rôle clef: Jeanne Deveaux fonda une école pour apprendre aux Noirs à lire et à écrire alors que John H. Deveaux fonda l'African Episcopal Church puis le Colored Tribune en 1875.

9 Les générations suivantes renforcèrent davantage les liens entre Haïti et Philadelphie en invitant les Noirs américains à s'établir en Haïti et à y construire des Églises protestantes: le pasteur Thomas Paul fut à l'origine de l'African Baptist Movement en Haïti, tandis que l'évêque James Theodore Holly joua un rôle considérable en y installant l'Église épiscopale. Cependant, l'assimilation y fut plus compliquée car ces 
libres de couleur, métis pour la plupart, membres de l'élite, fuyant le racisme ambiant des grandes villes de l'Est des États-Unis, refusaient de se faire gouverner par des officiels noirs.

10 Pour Ousmane Power-Greene, l'émigration d'un certain nombre d'AfricainsAméricains à Trinidad à la fin des années 1830, période faste de l'abolitionnisme et de la défense des droits de l'homme, doit être replacée dans un contexte plus large, celui d'une migration dans l'espace nord-américain vers Haïti, Trinidad et le Canada. Cette migration faisait débat dans la presse noire (entre Charles Ray et William Burley) et marquait la volonté des migrants de résister à la colonisation à destination du Liberia que certains Noirs libres considéraient comme un véritable projet de déportation. En 1839, 130 Noirs libres quittèrent Philadelphie pour Trinidad, attirés par les emplois qualifiés (mécanicien, charpentier, tonnelier), la possibilité de se faire instruire, ainsi que la promesse de percevoir une allocation de 25 \$. Or la réalité fut beaucoup moins plaisante: obligés de travailler sur des plantations, confrontés à la jalousie de la population locale, un tiers des migrants rentrèrent aux États-Unis dans les mois qui suivirent leur arrivée.

11 La communication de Nicolas Terrien (doctorant à l'université de Nantes), intitulée "Personnes de couleur libres et "masterless Caribbean" dans la période révolutionnaire (1790-1820)» s'est concentrée sur le concept du «masterless Caribbean " théorisé par Julius Scott dans The Common Wind: Afro-American Currents in the Age of the Haitian Revolution (2018). Selon Scott, il s'agit d'un nouvel ordre social défini par les boucaniers et pirates ayant fui l'Europe, hérité de la notion de " masterless men ", désignés par Christopher Hill dans The World Turned Upside Down comme des vagabonds et voleurs qui défiaient l'ordre féodal, des exclus contraints à l'errance. Perez Morales, dans No Limits to their Sway: Cartagena's Privateers and the Masterless Caribbean in the Age of Revolutions reprend cette vision d'un "monde souterrain de marrons, déserteurs et libres de couleur essayant continuellement d'échapper aux maitres et aux officiels » (p. 9). En étudiant les libres de couleur engagés dans des activités interlopes, Nicolas Terrien s'interroge sur ce groupe en se demandant s'il convenait de les considérer comme un groupe subversif ou si le masterless Caribbean pouvait faire référence à tout individu d'ascendance africaine circulant dans la Caraïbe avant 1830. Or, selon lui, les personnes de couleur libres étaient loin d'y former un groupe homogène et se différenciaient des esclaves marrons qui étaient reconnus par les autorités.

Romy Sanchez (membre du CNRS-IRhis Lille), a présenté le cas des libres de couleur cubains en exil au xIX siècle. Suite à l'indépendance d'un grand nombre de colonies espagnoles en 1824, Cuba, qui n'obtiendra son indépendance qu'en 1898, est le théâtre de nombreux mouvements visant à réclamer à la couronne davantage d'autonomie et de réformes. A partir des années 1830, nombre de ces réformistes subissent un exil forcé par les autorités impériales craignant une contagion d'indépendantisme. La plupart de ces proscrits ou exilés volontaires sont des créoles blancs, mais une minorité d'entre eux sont des libres de couleurs. Déportés hors de Cuba, ces individus fragilisés par la racialisation dont ils sont l'objet ne connaissent pas les mêmes parcours d'exil que les Blancs. Le consul espagnol s'est mis à surveiller cette population accusée de conspiration, sous l'influence supposée des abolitionnistes étatsuniens. Entre 1843 et 1844, 433 libres de couleur se retrouvent par exemple bannis du golfe du Mexique (au Brésil, au Mexique, et en Floride). Certains sont envoyés de force à Fernando Poo, île 
espagnole au large de la Guinée qui sert de Bagne. L'intervenante s'interroge donc sur la migration de ces libres de couleur : fut-elle contrainte ou forcée ? Relève-t-elle du bannissement ou de l'exil ? Si exil il y eut, ce dernier fut ségrégué, quand bien même les libres de couleurs se retrouvaient dans les mêmes circuits que les créoles blancs.

Céline Flory (chargée de recherches au CNRS-Mondes Américains, CIRESC) aborde le thème de "la maladie de la locomotion", cette mobilité des engagés africains en Guyane dans la seconde partie du xix siècle. Cette dernière communication porte audelà des bornes chronologiques choisies pour cette journée puisqu'elle se situe dans la période du post-esclavage. Après l'abolition de l'esclavage en Guyane française le 10 août 1848, la colonie connait une nouvelle migration de personnes de couleur venues d'Afrique, d'Inde et de Chine. Ainsi, de 1854 à 1857, 843 hommes et 41 femmes font le choix de quitter leur pays du littoral ouest-africain pour aller travailler plusieurs années en Guyane française dans le cadre réglementé de l'engagisme. Leurs contrats prennent en charge les frais de transport, de nourriture, de logement, un faible salaire ainsi que l'assurance d'un rapatriement en fin de contrat. Cette nouvelle population engagée se mêle ainsi à celle des captifs qui, déjà présents en Amérique, se font alors racheter. Or, quelques semaines ou quelques mois après leur arrivée, ils commencent à remettre en cause leurs contrats : le type d'emploi, le temps d'engagement, le montant du salaire, le lieu d'engagement, ou le type d'engagisme (ils pensaient par exemple travailler pour le gouvernement français et non pour des particuliers). Se trouvant rapidement confrontés à des conditions de vie et de travail aux antipodes de celles originellement présentées par les recruteurs et à la surdité des autorités coloniales visà-vis de leurs réclamations, nombre de ces engagés décidèrent de migrer à nouveau, de façon illégale cette fois, vers des territoires voisins non français comme la Guyane anglaise (Guyana) ou la Guyane néerlandaise (Surinam). Ces migrants se trouvent d'ailleurs une nouvelle identité, prenant des noms venus du monde maritime, comme Perroquet, Canot, Liverpool, Jamaïque, Bouteille de bière, Bouchon, ou Couteau. Les proportions de migrants sont telles que le gouverneur leur attribue une maladie, celle de la «locomotion ». Si cette naturalité lui permet de dédouaner État et employeurs de toute responsabilité dans ces évasions criminelles (ils quittaient la colonie avant la fin de leur contrat), elle révèle en creux tant la fonction première souhaitée de l'engagisme (immobiliser la main d'œuvre) que son échec. Par ailleurs, cette re-migration met en exergue non seulement la pleine conscience qu'ont ces engagés africains de leur liberté, mais l'incapacité de la société guyanaise à leur reconnaître celle-ci et à se montrer de fait société post-esclavagiste.

\section{INDEX}

Thèmes : Actualité de la recherche 
AUTEUR

ANNE-CLAIRE FAUCQUEZ

Université Paris 8 